\title{
The effect of deep shoulder infections on patient outcomes after arthroscopic rotator cuff repair: a retrospective comparative study
}

\author{
Kivanc Atesok ${ }^{1,2}$ \\ Peter MacDonald 1 \\ Jeff Leiter ${ }^{1}$ \\ Sheila McRae 1 \\ Mandip Singh ${ }^{1}$ \\ Greg Stranges ${ }^{1}$ \\ Jason Old ${ }^{1}$ \\ 1 Section of Orthopaedic Surgery, PanAm Clinic, \\ University of Manitoba, Winnipeg, Manitoba, Canada \\ 2 Department of Orthopaedic Surgery, University of \\ Alabama at Birmingham, Birmingham, Alabama, USA
}

Corresponding author:

Kivanc Atesok

Department of Orthopaedic Surgery,

University of Alabama at Birmingham

510 20th Street South, FOT260

Birmingham, AL, 35294 USA

Tel.: +1 2059097843

E-mail: Katesok@uabmc.ca

\section{Summary}

Introduction: The purpose of this study was to evaluate the effects of deep shoulder infections after RCR on patient outcomes.

Methods: A retrospective chart review was conducted involving all patients with deep shoulder infections after arthroscopic RCR (study group). Another group of patients who were matched with the study group by age, gender and rotator cuff tear size, and did not develop deep shoulder infections after arthroscopic RCR were randomly identified (control group). The two groups were compared in terms of time to start physiotherapy, shoulder function, and delay in return to work.

Results: There were 10 patients in each group. The mean time to start physiotherapy after surgery was 145.3 $(S D=158.8)$ days for the study group and $\mathbf{4 0 . 0}$ $(S D=13.7)$ days for the control group $(p=.051)$. The average forward elevation of the operated shoulder was $133(S D=33.4)$ degrees for the study group, and $172(S D=12.0)$ degrees for the control group $(p=0.003)$. The average time to return to work at preoperative level was $\mathbf{5 . 6}$ months for the study group and 3 months for the control group.
Conclusion: Deep shoulder infections after RCR significantly impedes time to start physiotherapy, shoulder function, and patients' ability to return to work.

Level of evidence: III b [retrospective comparative (case-control) study].

KEY WORDS: rotator cuff tear, arthroscopic cuff repair, post-operative complications, deep shoulder infections, patient outcomes.

\section{Introduction}

Rotator cuff repair (RCR) is one of the most frequently performed orthopaedic procedures, and arthroscopic repair has become the procedure of choice for the last two decades, with good or excellent clinical outcomes in approximately $90 \%$ of patients ${ }^{1-4}$. The data obtained from the National Hospital Discharge Survey and the National Survey of Ambulatory Surgery indicate that the number of rotator cuff repairs increased $141 \%$ between 1996 to 2006 in the United States ${ }^{2}$. Interestingly, the number of arthroscopic repairs increased by $600 \%$, whereas open repairs increased by only $34 \%$ during the same period ${ }^{2}$. Due to the exponential increase in the number of arthroscopic RCRs, complications may be encountered more frequently ${ }^{1}$. Postoperative deep shoulder infections after arthroscopic RCR appear to be a rare complication, with a reported incidence of $8.5 / 1000^{5}$. However, this condition can be permanently disabling for the patient, with devastating effects on functional outcomes. Studies indicate that post-RCR deep infections may result in abscess formation, osteomyelitis, post-infectious glenohumeral arthritis, and loss of the soft tissue envelope ${ }^{1,6-8}$. Although some of the studies in which open or mini-open RCR techniques were used, confirm poor outcomes as a result of post-operative infections ${ }^{1,6-8}$, no studies to date have reported deleterious effects of deep infections after arthroscopic RCRs on functional outcomes, time to return to work, or time to start physiotherapy in comparison with a control group.

The purpose of this investigation was to evaluate the effects of deep shoulder infections after arthroscopic RCR on patient outcomes and compare the results with those of a matched group of patients who did not develop infections after arthroscopic RCR. 
The effect of deep shoulder infections on patient outcomes after arthroscopic rotator cuff repair: a retrospective comparative study

\section{Materials and methods}

The current study meets the ethical and scientific standards of the Muscles, Ligaments and Tendons Journal as defined by Padulo et al. ${ }^{9}$ in "Basic principles and recommendations in clinical and field science research: 2016 Update."

After obtaining approvals from the Institutional Review Board and the Regional Health Authority, a computerized search based on International Classification of Diseases (ICD) codes and keywords was conducted. All primary arthroscopic RCRs performed both ambulatory and inpatient between 2010 and 2016 in the department were captured. Patients treated with arthroscopic mini-open procedures, and those with a previous history of open shoulder surgery were excluded from the study. Cases of post-arthroscopic $\mathrm{RCR}$ infections requiring additional surgery and longterm intravenous (IV) antibiotic treatment within 8 weeks of the index procedure were identified and defined as the infection (study) group. A detailed review of archived patient charts, electronic medical records, and $\mathrm{MRI}$ reports was performed to determine the incidence of infections, patient demographics, rotator cuff tear size, operating surgeon, post-operative treatment and rehabilitation, shoulder range of motion (ROM), and return to work. Based on collected data, a group of patients who matched those in the study group in terms of age, gender, tear size, and surgeon in charge were randomly chosen and defined as the control group.

\section{Definition of study parameters}

Tear size

The data about tear size was collected from the MRI reports and noted within one of the four categories based on the greatest tear dimension: small $(0-1 \mathrm{~cm})$, medium (1-3 cm), large $(3-5 \mathrm{~cm})$, and massive $(>5$ $\mathrm{cm})$, as described by DeOrio and Cofield ${ }^{10}$. For the purpose of this study, complete and partial tears were not separately categorized based on the fact that during arthroscopic RCR, all partial tears were debrided and converted into complete tears before repair.

\section{Time to start physiotherapy}

All the patients were routinely referred to physiotherapy after arthroscopic RCR to start between fourth to sixth post-operative weeks. Physiotherapists regularly reported to the orthopaedic surgeons regarding their respective patients' progress in rehabilitation, and this data was included in electronic medical records or archived charts with the physiotherapy start date.

\section{Shoulder range of motion}

Forward shoulder elevation was chosen as the parameter for comparison because this was the only consistently measured data regarding shoulder ROM from both groups due to the retrospective nature of the present study.

\section{Selection of control group patients}

All infection group patients were male. Hence, a pool of 40 male patients within the same age range as the study group who were operated on by the same surgeons was randomly chosen from the patients captured using the computerized search described above. Out of these 40 patients, the first ten patients whose tear sizes matched with those of the patients in the study group were selected sequentially, moving from more recent to more remote procedure dates. The two groups were compared in terms of physiotherapy start date, shoulder ROM specified as forward elevation, and time to return to work.

\section{Other documented variables}

At the first outpatient visit, all the patients were routinely given a questionnaire regarding additional illnesses, medications, and smoking status. In addition, patients in the study group were also documented in terms of culture results, antibiotic treatment, and additional surgeries for shoulder infection.

\section{Statistical analysis}

Descriptive data was generated for both the study and control groups. Continuous variables were compared between groups using a series of independent t-tests and categorical data was compared using Fisher's exact test. Significance for all comparisons was alpha $=0.05$.

\section{Results}

There were 2,249 primary arthroscopic RCRs performed in patients who have failed conservative therapy between 2010 and 2016 at our institution ${ }^{11}$. A total of 10 patients developed deep shoulder infections after arthroscopic RCR. All the patients in the infection group were male, with a mean age of $55.6 \pm 8.4$, and the mean follow-up time was $6.3 \pm 2.1$ months. In the infection group, two patients had small, six patients had medium, and two patients had large size rotator cuff tears. Table I displays the patient demographics for the infection group.

The overall post-arthroscopic RCR deep infection rate at our institution was $0.44 \%$. The infections were treated with an average of 1.6 surgical debridements (range: 1 to 3 ) with the first debridement occurring on average 22.9 days (range: 9 to 68 days) after the index procedure. All debridements were performed arthroscopically, except for that of one patient, who had two arthroscopic debridements followed by one open debridement due to development of osteomyelitis (Fig. 1). Only in one patient the RCR appeared intact during arthroscopic debridement and no suture material or anchors were removed. The RCRs in all other infected group patients were found to be disrupted, and suture material and anchors were removed during arthroscopic irrigation and debridement. Microbiological evaluation revealed positive culture results in all except for one patient, who was 
K. Asetok et al.

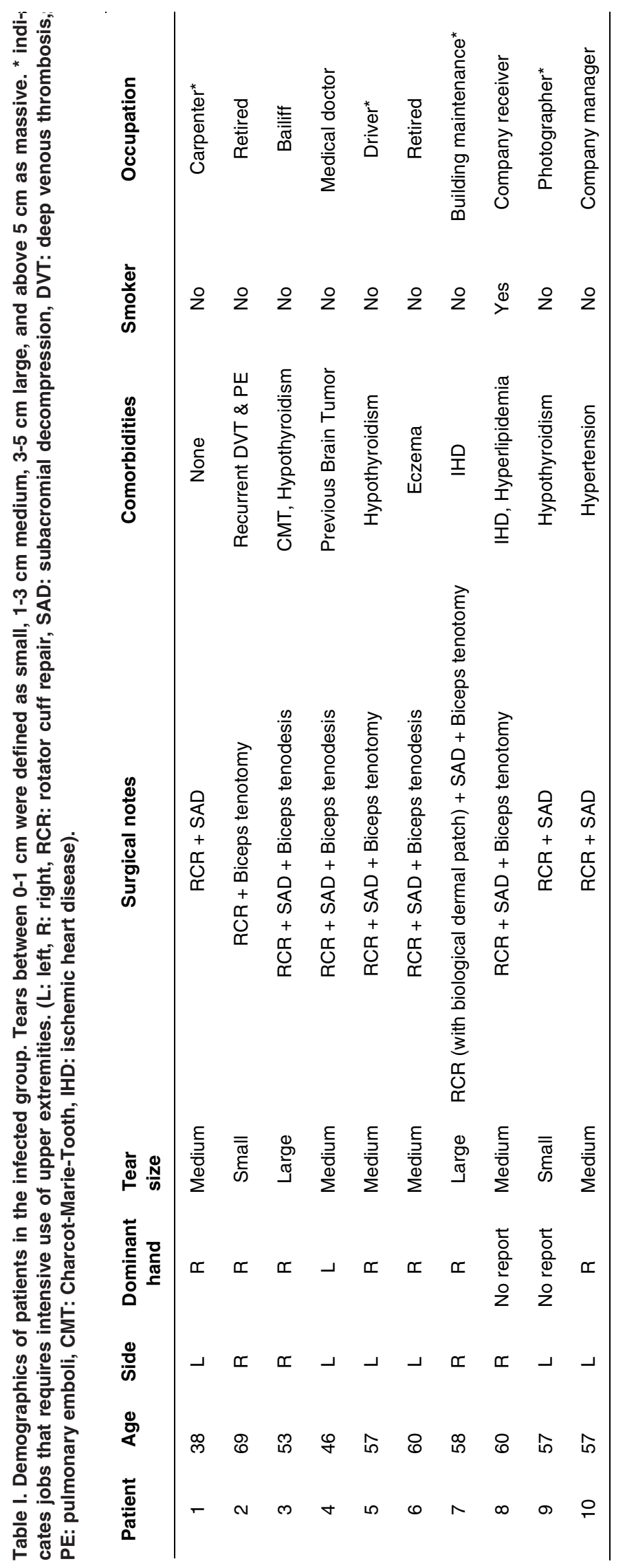


The effect of deep shoulder infections on patient outcomes after arthroscopic rotator cuff repair: a retrospective comparative study
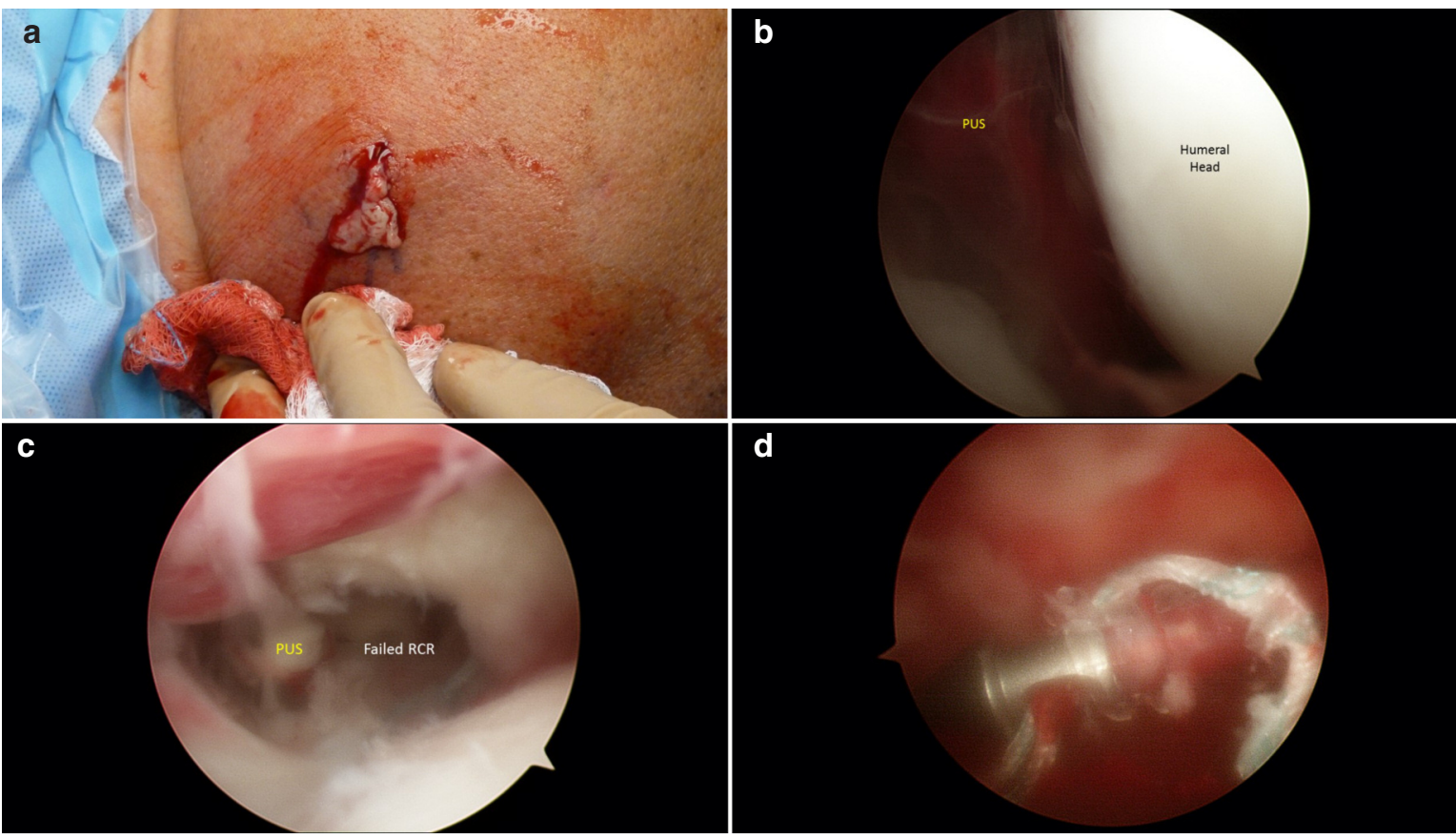

Figure 1 a-d. Illustrations from a 53-year-old male patient who underwent arthroscopic irrigation and debridement due to deep shoulder infection 3 weeks after arthroscopic RCR. a. There was profuse drainage of thick PUS from the opened arthroscopy portal; b. arthroscopic images also revealed substantial pus in gleno-humeral joint; c. rotator cuff repair from the index procedure appeared to be failed with pus and reactive synovitis all around the torn cuff; $d$. loosened suture material and anchor was removed. After aggressive debridement of the infected tissues, the joint is washed profusely using a minimum of 10 liters of fluid, with the last liter containing 100,000 units of bacitracin.

started on oral antibiotics by his family physician before he was seen by the operating surgeon. Seven patients had growth of single microorganisms including Staphylococcus aureus (5), Propionibacterium acnes (1), and Phaeoacremonium parasiticum (1). Culture results from each of the remaining two patients showed growth of two microorganisms: Propionibacterium acnes with Staphylococcus saccharolyticus, and Staphylococcus epidermidis with Staphylococcus saccharolyticus. All the patients were treated with long-term IV antibiotics delivered through a peripherally-inserted central catheter (PICC) line, and the infections were eradicated. However, two patients received additional oral antibiotic treatment and were scheduled for longer follow-up due to development of osteomyelitis of the humeral head.

The control group was comprised of 10 patients who underwent arthroscopic RCR during the same period as the infection group patients and did not develop any postoperative infections (Tab. II). Although there was no statistical difference between the two groups in terms of age, gender, rotator cuff tear size, or follow-up time, there were significantly more patients with comorbidities in the infection group than in the control group (nine vs four, respectively, $p=0.03$ ). One patient in the control group received intraoperative IV antibiotic prophylaxis compared with the infection group where 2 patients were given IV antibiotics intraoperatively during the index arthroscopic RCR.
The outcomes in the infection group were worse in terms of forward flexion, time to start physiotherapy, and return to work (Tab. III). In the infection group, one patient could not work again after the original $\mathrm{RCR}$, three patients could go back to light duties after an average of nine months, and the rest started working at their preoperative level after an average of 5.6 months. In addition to the two patients with osteomyelitis, three patients in the infection group were noted as permanently disabled due to severe stiffness, decreased ROM, and pain; one of these patients was already scheduled for reverse shoulder replacement at the last follow-up. In the control group, two patients could start working in light duties after three post-operative months, and the rest of the patients started working at the preoperative level at an average of three months after the RCR. No patients had permanent disabilities or complaints in the control group at their last follow-up visit.

\section{Discussion}

Deep infections of the shoulder after arthroscopic $\mathrm{RCR}$ are uncommon. The results of the present study indicate an overall deep infection rate of $0.44 \%$ that appears to be lower than the rate of $0.85 \%$ reported by Pauzenberger et al. ${ }^{5}$. This difference in rate of infection can possibly be attributed to the difference be- 


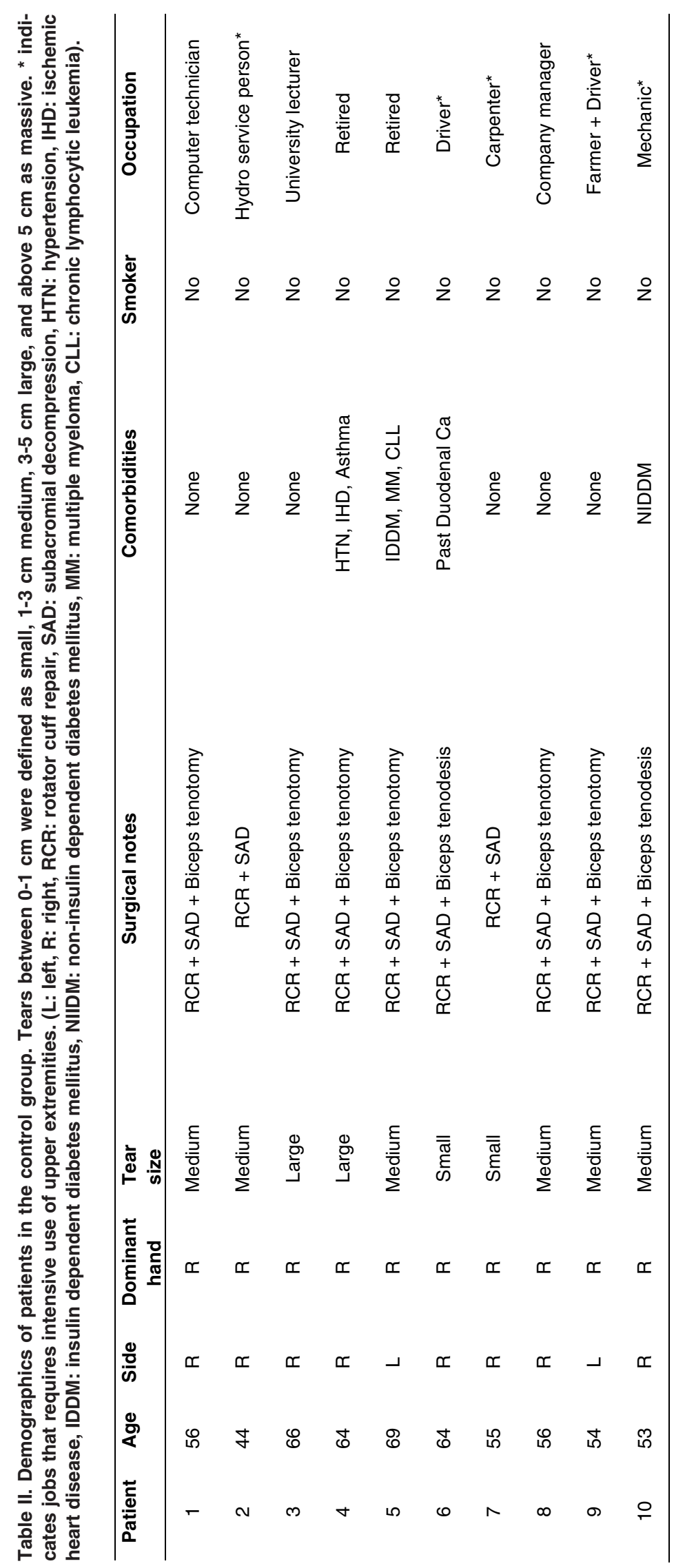


The effect of deep shoulder infections on patient outcomes after arthroscopic rotator cuff repair: a retrospective comparative study

Table III. Comparison of age, follow-up time, time to start physiotherapy, and forward shoulder elevation between infection and control groups. Please note that the large difference in mean time to start physiotherapy between the two groups (105.8 days) appeared not statistically significant due to small patient numbers. However, it is undisputable that a delay of such magnitude may cause clinically significant differences in functional outcomes.

\begin{tabular}{lccc}
\hline & Infection & Control & \\
\hline Mean age (years) & $55.6 \pm 8.4$ & $58.1 \pm 7.5$ & $P=0.492$ \\
Mean follow-up (months) & $6.3 \pm 2.1$ & $4.8 \pm 2.8$ & $P=0.192$ \\
Time to start physiotherapy (days) & $145.8 \pm 158.8$ & $40.0 \pm 13.7$ & $P=0.051$ \\
Forward shoulder elevation (degrees) & $133 \pm 33.4$ & $172 \pm 12$ & $P=0.003$ \\
\hline
\end{tabular}

tween the number of patients included in the present study and the study from Pauzenberger et al. ${ }^{5}(2,249$ vs 3,294, respectively).

Published literature shows that the most commonly isolated microorganisms in patients with deep shoulder infections after RCR include Propionibacterium acnes, coagulase-negative staphylococci (e.g., Staphylococcus epidermidis and Staphylococcus saccharolyticus), and Staphylococcus aureus $1,6,7,12,13$. The data from the present study are comparable to previously published reports; eight out of nine patients from whom a causative microorganism could be isolated were infected with one or two of the above mentioned pathogens.

The current investigation revealed significantly worse outcomes in patients who had deep shoulder infections after arthroscopic RCR in terms of forward shoulder elevation and clinically important delays in time to start physiotherapy and return to work. Previously, Kwon et al. ${ }^{6}$ reported an active shoulder elevation of $119^{\circ}$ in their study, which included 12 patients with deep shoulder infection at a mean follow-up time of 37.5 months. The infected patients in the present study had an average shoulder elevation of $133^{\circ}$ at a mean follow-up time of 6.3 months. Better ROM measured in our infection group, especially after a shorter time post-operatively, may be explained by the differences in surgical techniques. In our study, all the index RCRs and first debridement procedures were performed arthroscopically. However, Kwon et al. ${ }^{6}$ used open or mini-open RCR techniques as the index procedure, and all the debridement procedures were performed with open surgery.

Post-operative physiotherapy is an important determinant of clinical success after $\mathrm{RCR}^{14,15}$. Evidence indicates that early ROM exercises lead to accelerated recovery from postoperative joint stiffness and increased forward elevation following arthroscopic $\mathrm{RCR}^{16}$. To the best of our knowledge, no studies to date have reported on the delay in starting physiotherapy due to deep shoulder infections after arthroscopic RCR. In the present study, the patients were rehabilitated based on a conventional post-RCR physiotherapy protocol, which allows only pendulum shoulder motion until up to six weeks postoperatively.
During the sixth postoperative week, all the patients routinely started gradual ROM and strengthening exercises with expert physical therapists. The delay in starting physiotherapy was found to be longer than three months for the infection group (105 days). Although this difference between the two groups in the mean time to start physiotherapy was not calculated to be statistically significant, failure to start shoulder exercises until almost five months postoperatively (145 days) is a substantial delay.

Return to work after arthroscopic RCR depends on the type of work the patient does, in addition to other variables such as age, gender, and in some cases workers' compensation ${ }^{17-19}$. In a study including 365 patients, Collin et al. ${ }^{17}$ reported that approximately $20 \%$ of the patients were unable to resume normal activity at six months after arthroscopic RCR. The authors noted that persistent limitation at six months was associated with female gender, heavy manual work, and the presence of persistent postoperative bursitis. In the current study, each group included two retired patients. There were four patients in the infection group and five patients in the control group whose jobs required intensive use of the upper extremities (Tabs 1,2). The only patient in the infected group who could not return to his previous job used to work in building maintenance. In the infection group, three patients could resume only light duties after an average of nine months (carpenter, driver, company receiver) and four patients could return to full duties at an average of 5.6 months (bailiff, medical doctor, photographer, and manager). Both patients in the infection group who developed osteomyelitis (medical doctor and bailiff) and another patient (company manager) who had residual disability due to limitations in ROM, could return to full duties at work. In the control group, the only two patients who could return to light duties within three months were a carpenter and a driver. The other three patients in the control group whose jobs required using the upper extremities intensively could return to their previous jobs resuming full duties without notable delays. These results indicate that deep shoulder infections after arthroscopic RCR impede patients' return to work regardless of their occupation. It is conceivable that arthroscopic or 
open irrigation and debridement, along with long-term IV antibiotic treatment to eradicate deep shoulder infections, will almost certainly cause important delays for patients' resuming duties at work and decrease their quality of life.

Another point worth discussing is the possibility of developing devastating complications due to deep shoulder infection, such as osteomyelitis and abscess formation ${ }^{1,8}$. In a series of 13 patients from Mirzayan et al. ${ }^{8}$ with chronic deep infections following open RCR, 11 patients had osteomyelitis of the humeral head, the glenoid, and/or the clavicle and the acromion. Two patients had no osteomyelitis but had a subdeltoid abscess, and seven patients required a rotational flap to allow for soft tissue coverage. In the present study, there were two patients in the infection group who developed osteomyelitis of the humeral head as a result of deep shoulder infections after arthroscopic RCR. Interestingly, there were three patients in the infection group who had biceps tenodesis in addition to RCR and subacromial decompression, and two of those patients were also the ones who developed osteomyelitis.

There are a few limitations in the present investigation that are inherent to retrospective data collection for rare occurrences, such as deep shoulder infections after arthroscopic RCR. There were only 10 patients in the infection group, and this may be the primary reason why substantial differences, such as 105 days of delay in the start of physiotherapy, were found not to be statistically significant. Additionally, functional outcomes were not uniformly recorded for each patient, and the only consistently measured ROM parameter was forward elevation. Hence, available data did not include validated shoulder scores. On the other hand, the use of a control group with patients who matched those in the infected group in terms of age, gender, tear size, and surgical approach was the main strength of this investigation.

\section{Conclusion}

Deep shoulder infections after arthroscopic RCR substantially impede time to start physiotherapy, shoulder function, and patients' ability to return to work. The detrimental effects of post-RCR deep shoulder infections on the healthcare system require further attention and a methodological approach to minimize negative consequences.

\section{Acknowledgement}

The Authors would like to thank to Dr. Randa Berdusco, Dr. James Dubberley, Dr. Jonathan Marsh, Dr. Ted Tufescu, Ms. Carmen Bailey and Ms. LeeAnne Gullett for their help in data acquisition and completion of this project.

\section{Conflict of interests}

The Authors declare that they have no conflict of interests regarding the publication of this paper.

\section{References}

1. Atesok K, MacDonald P, Leiter J, et al. Postoperative deep shoulder infections following rotator cuff repair. World J Orthop. In press. 2017.

2. Colvin AC, Egorova N, Harrison AK, et al. National trends in rotator cuff repair. J Bone Joint Surg Am. 2012;94(3):227233.

3. Flanagin BA, Garofalo R, Lo EY, et al. Midterm clinical outcomes following arthroscopic transosseous rotator cuff repair. Int J Shoulder Surg. 2016;10(1):3-9.

4. Ikemoto RY, Murachovsky J, Nascimento LG, et al. Arthroscopic repair of small and medium tears of the supraspinatus muscle tendon: evaluation of the clinical and functional outcomes after two years of follow-up. Rev Bras Ortop. 2015;47(4):436-440.

5. Pauzenberger L, Grieb A, Hexel M, et al. Infections following arthroscopic rotator cuff repair: incidence, risk factors, and prophylaxis. Knee Surg Sports Traumatol Arthrosc. 2016 Jun 24. [Epub ahead of print]. PMID: 27342982.

6. Kwon YW, Kalainov DM, Rose HA, et al. Management of early deep infection after rotator cuff repair surgery. J Shoulder Elbow Surg. 2005;14(1):1-5.

7. Athwal GS, Sperling JW, Rispoli DM, et al. Deep infection after rotator cuff repair. J Shoulder Elbow Surg. 2007;16(3): 306-311.

8. Mirzayan R, Itamura JM, Vangsness CT Jr, et al. Management of chronic deep infection following rotator cuff repair. J Bone Joint Surg Am. 2000;82-A(8):1115-1121.

9. Padulo J, Oliva F, Frizziero A, Maffulli N. Muscles, Ligaments and Tendons Journal - Basic principles and recommendations in clinical and field science research: 2016 update. MLTJ. 2016;6(1):1-5.

10. DeOrio JK, Cofield RH. Results of a second attempt at surgical repair of a failed initial rotator-cuff repair. J Bone Joint Surg Am. 1984;66(4):563-567.

11. Abdul-Wahab TA, Betancourt JP, Hassan F, et al. Initial treatment of complete rotator cuff tear and transition to surgical treatment: systematic review of the evidence. Muscles Ligaments Tendons J. 2016;6(1):35-47.

12. Settecerri JJ, Pitner MA, Rock MG, et al. Infection after rotator cuff repair. J Shoulder Elbow Surg. 1999;8(1):1-5.

13. Vopat BG, Lee BJ, DeStefano S, et al. Risk Factors for Infection After Rotator Cuff Repair. Arthroscopy. 2016;32 (3):428-434.

14. Ross D, Maerz T, Lynch J, et al. Rehabilitation following arthroscopic rotator cuff repair: a review of current literature. J Am Acad Orthop Surg. 2014;22(1):1-9.

15. Oliva F, Piccirilli E, Bossa M, et al. I.S.Mu.L.T - Rotator Cuff Tears Guidelines. Muscles Ligaments Tendons J. 2016; 5(4):227-263.

16. Chang KV, Hung CY, Han DS, et al. Early Versus Delayed Passive Range of Motion Exercise for Arthroscopic Rotator Cuff Repair: A Meta-analysis of Randomized Controlled Trials. Am J Sports Med. 2015;43(5):1265-1273.

17. Collin $\mathrm{P}$, Abdullah A, Kherad O, et al. Prospective evaluation of clinical and radiologic factors predicting return to activity within 6 months after arthroscopic rotator cuff repair. J Shoulder Elbow Surg. 2015;24(3):439-445. 
The effect of deep shoulder infections on patient outcomes after arthroscopic rotator cuff repair: a retrospective comparative study

18. Lazarides AL, Alentorn-Geli E, Choi JH, et al. Rotator cuff tears in young patients: a different disease than rotator cuff tears in elderly patients. J Shoulder Elbow Surg. 2015; 24(11):1834-1843.
19. De Moraes VY, Godin K, Dos Santos JB, et al. Influence of compensation status on time off work after carpal tunnel release and rotator cuff surgery: a meta-analysis. Patient Saf Surg. 2013;7(1):1. 